\title{
Application practice of Oriental design strategy in interior space design
}

\author{
Yihui $\mathrm{Du}^{1}$, Lili Peng ${ }^{1}$, Zijing Zhang ${ }^{1}$ and $\mathrm{Yi} \mathrm{Li}^{1}{ }^{1, *}$ \\ ${ }^{1}$ Architectural and Urban Planning Department, Chongqing College of Architecture and Technology, 401331, China
}

\begin{abstract}
Oriental design is based on Oriental cultural tradition and philosophy. After merging with the essence of modern design, it has gained new vitality and constantly meets the spiritual and material needs of modern people. This paper mainly summarizes the design strategies from three aspects: element minimalism, light and shadow inheritance and meditation practice. Combined with the team practice case analysis, this paper hopes to provide help for other designers' localization design thinking. It is hoped that designers can establish cultural confidence based on their understanding of Oriental culture and philosophy, and naturally integrate Oriental Spirit into future design.
\end{abstract}

\section{Introduction}

Oriental design is a design philosophy, theoretical system and practical activity rooted in oriental culture and philosophy. It is a design mode and concept that consciously occurs and constantly advances in design practice to meet the aesthetic and functional needs of modern society.

Based on thousands of years of Oriental civilization, Oriental design adheres to the design concept of combining craftsmanship beauty, natural beauty and realm beauty, and combining life cultivation and social function. It emphasizes to give full play to the nature of things to the greatest extent, and takes the freedom between things and people, and the mutual service between things and people as the highest design criterion [1-3].

After the reform and opening up, Chinese interior design has experienced the process from learning from the west to looking for itself, and now it has formed a process of cultural consciousness and tolerance of western design. Chinese designers should be based on their own cultural confidence, no longer deliberately copy and reproduce the classical tradition, and naturally integrate the Oriental Spirit into the design. Only in this way can Chinese design be localized.

In the actual interior design projects, the author's interior design team widely adopts Oriental design techniques, inherits the essence of Oriental traditional culture and philosophy, applies advanced modern design technology, combines the material and spiritual needs of contemporary people, and strives to explore the development road of Oriental design in line with the development requirements of the times, so as to make Chinese design influence or lead the world [3-6].

\section{Oriental Design Strategy}

\subsection{Minimalist elements and Painstaking investigation}

The literati of all ages in our country are all masters of showing off literary skill. In the space design, we use black ink and white paper, the stroke and painting, with the most concise technique, the most clean tone, to create the quality of space. Then designers use fewer elements to present a more humanistic artistic conception of space.

Using different perspectives, we can break the boundary between the East and the West from the concept of "nothing" and "emptiness". With very few elements and minimalist techniques, the design strategy of getting rid of the complexity and hitting the heart directly conforms to the imagination of Oriental design for space, and does not lose the style of modern design [6].

\subsection{Light and shadow inheritance, harmonious coexistence}

In the traditional oriental philosophy, it advocates the harmonious coexistence of man and nature, and does not try to transform nature. Respect is the best policy. In interior space design, light and shadow is the best gift given to the host by nature. Complementary light and shadow can produce the possibility of multiple sources, and can build a transparent, translucent and opaque, or reflective and refractive space.

Light makes space produce a kind of uncertain character, shaping a short, immediate feeling and experience in the travel time. With the moving of light, it

\footnotetext{
" Corresponding author: cqfdc2008@163.com
} 
not only enriches the changes of space, but also makes time flow repeatedly.

\subsection{Meditation practicing, and the state of quietness}

In oriental culture and philosophy, "meditation" is the only way to practice. Oriental design is rooted in the soil of Oriental philosophy and oriental traditional culture. Designers must take root in life, let culture nourish and history moisten their own ideas, and carry out "cultural excavation" of design regions and places.

What Oriental design brings us is a kind of "Quietness", a kind of quietness to experience and interpret life, and the real design comes from life [7]. Quietness is a state, a supreme state of life. Although the human "core", but the appropriate space can help to "touch the scene".

\section{Design Practice}

\subsection{Case 1: The design scheme of a residence in Chongqing}

Customer positioning: the master of the age of no doubt, the pursuit of quality, low luxury, elegant quality of life. In the design, the technique of line drawing is adopted, and the most concise technique, the cleanest tone and fewer elements are used to present a more humanistic and artistic space.

In the design of guest restaurant and tea house, the style of mountain, the flexibility of water pattern, traditional Chinese carving and other elements are used to create a poetic ancient space.

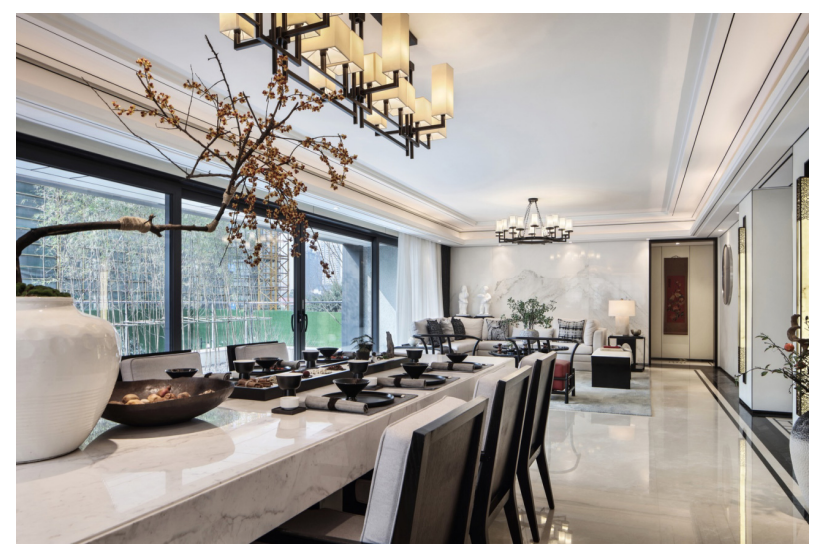

Figure 1. Real view of guest and restaurant.

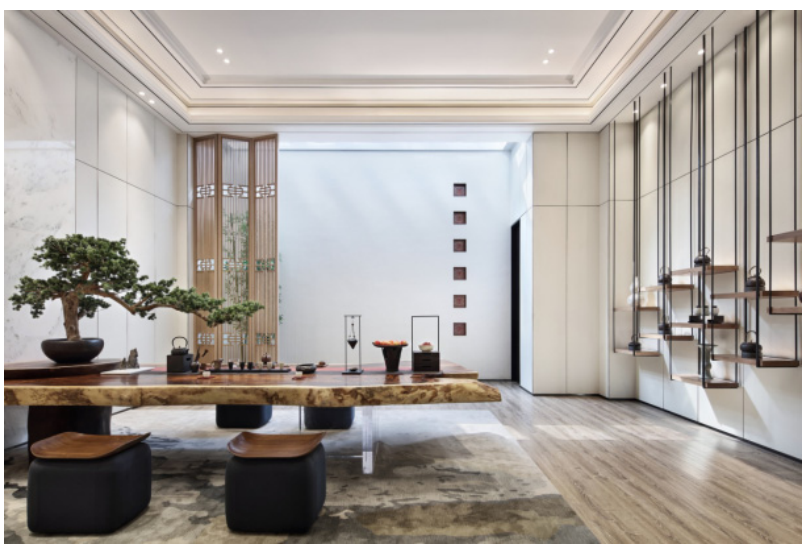

Figure 2. Real view of guest and restaurant.

"Thin stone logs together, graceful erecting does not change four spring". The corridor design adopts the combination of large area blank and traditional carving, one black and one white, giving the space elegant and

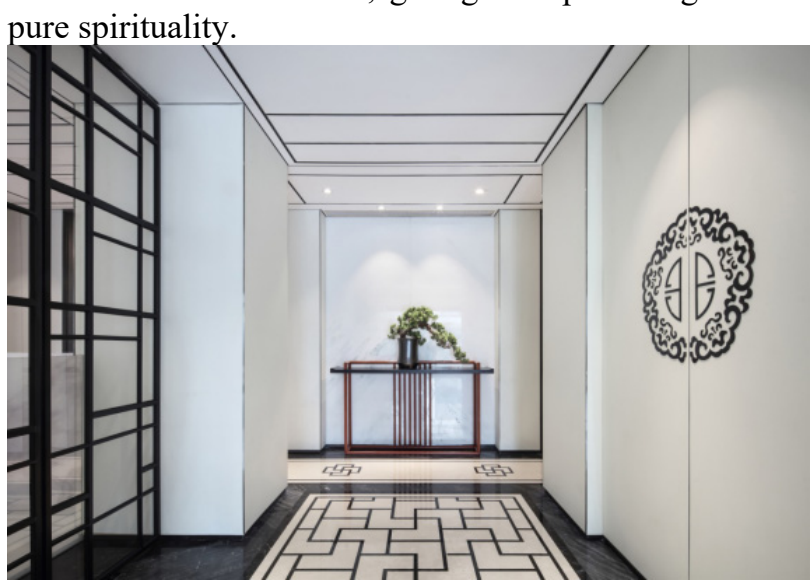

Figure 3. Real view of corridor 1 .

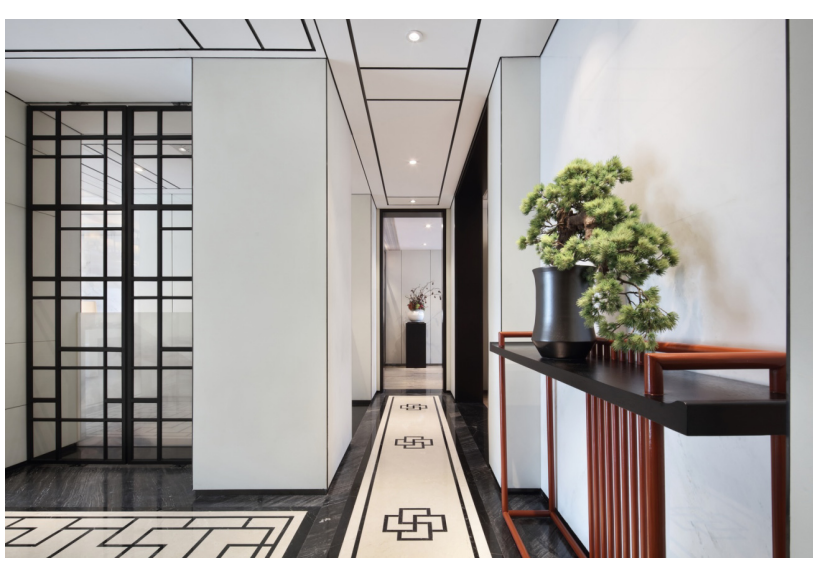

Figure 4. Real view of corridor 2.

The design of living room is mainly based on reduction, and every part of it is being deliberated, emphasizing the visual characteristics of the picture, which is closer to the style of Song people in terms of cultural roots. 


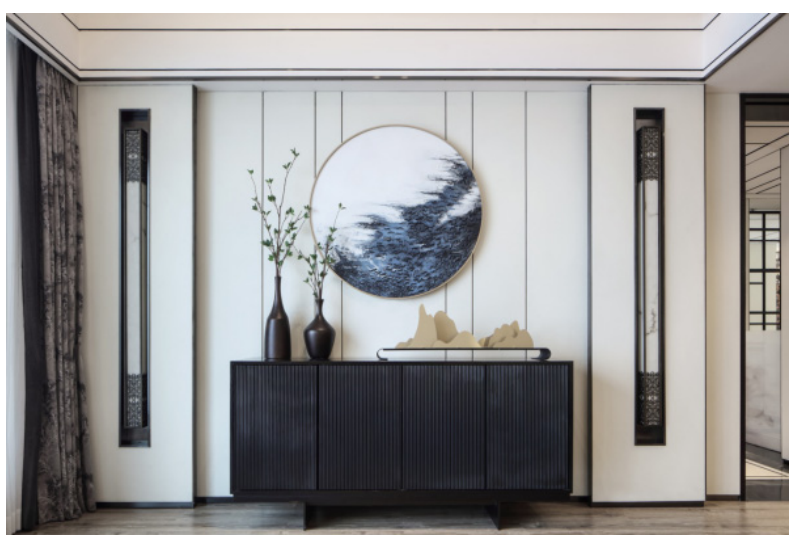

Figure 5. Real view of corridor 1.

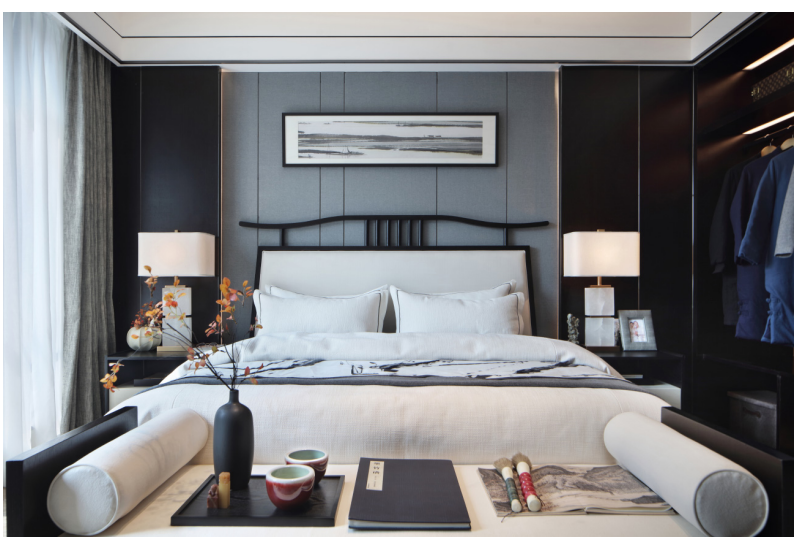

Figure 6. Real view of corridor 2.

\subsection{Case 2: The reconstruction scheme of a sales center in Kunming}

Project background: the facade of the building is made of all glass with strong permeability.

Kunming enjoys the reputation of "Spring City", with spring like seasons and abundant sunshine. Due to its high altitude and strong ultraviolet radiation, the average annual sunshine can reach 2480 hours. When "good permeability" meets "sufficient light", how to avoid its possible "light pollution"?

The design adopts the partition with a sense of form to isolate the direct sunlight. By controlling the relationship between angle and density, it skillfully controls the illumination of light, and presents a changeable effect in different angles and time periods.

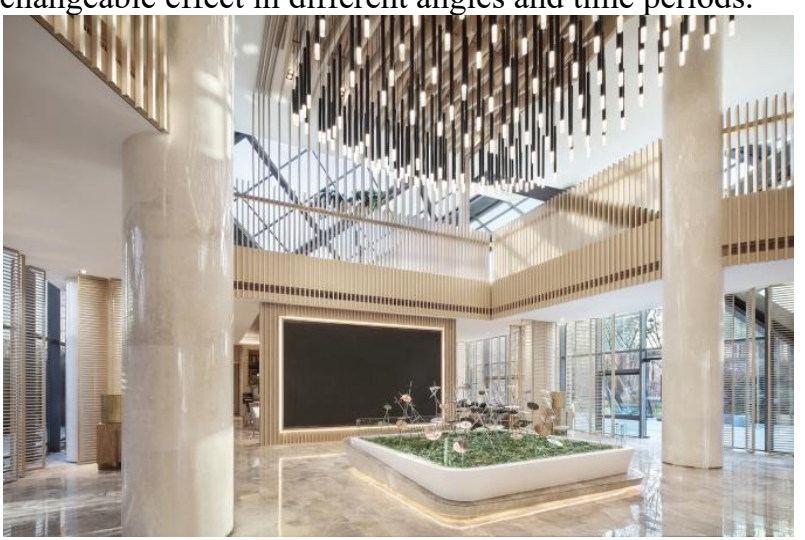

Figure 7. Real view of the lobby.

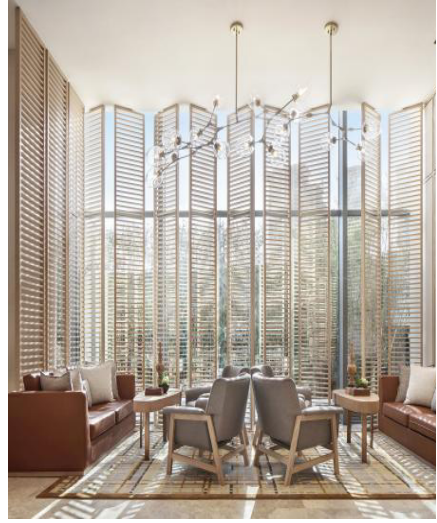

Figure 8. Real view of VIP negotiation area.

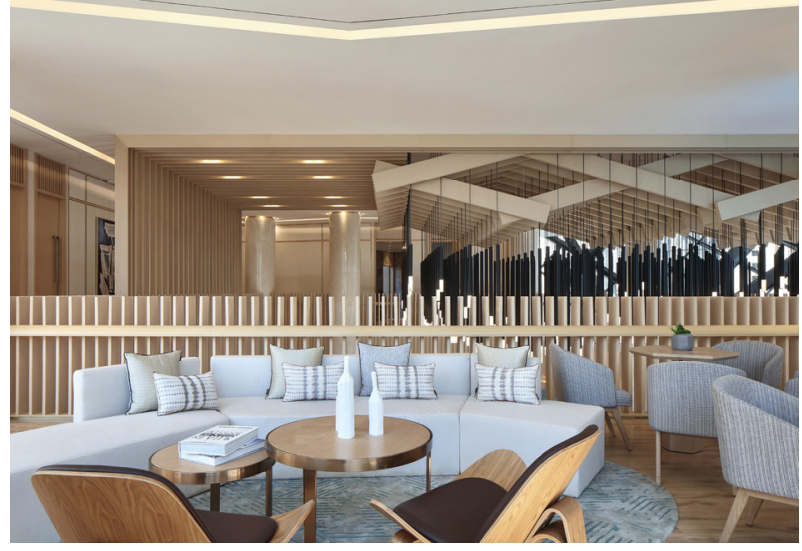

Figure 9. Real view of rest area.

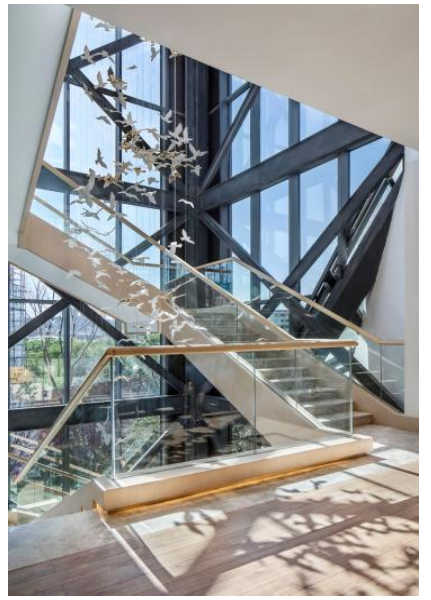

Figure 10. Real view of stairs.

\subsection{Case 3: The reconstruction scheme of a club in Chengdu}

Project background: the club reconstruction project is located in Chengdu, a city of $\mathrm{Ba}$ shu with strong historical background but with its own style. Tea house, folk art and face changing are the cultural symbols of the city.

The design concept adopted in the transformation scheme is: Meditation is a kind of practice, quiet is luxury, from simplicity to luxury, clean is technique, quiet is realm! 


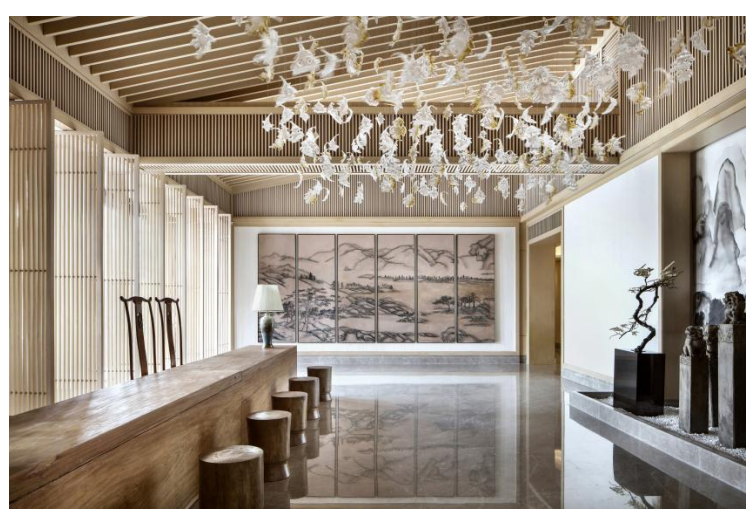

Figure 11. Real view of the hall 1.

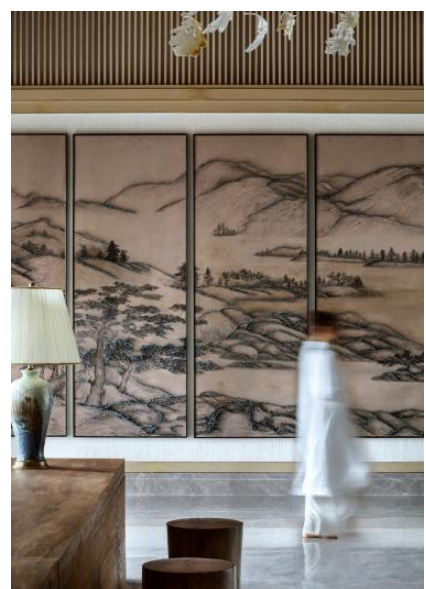

Figure 12. Real view of the hall 2.

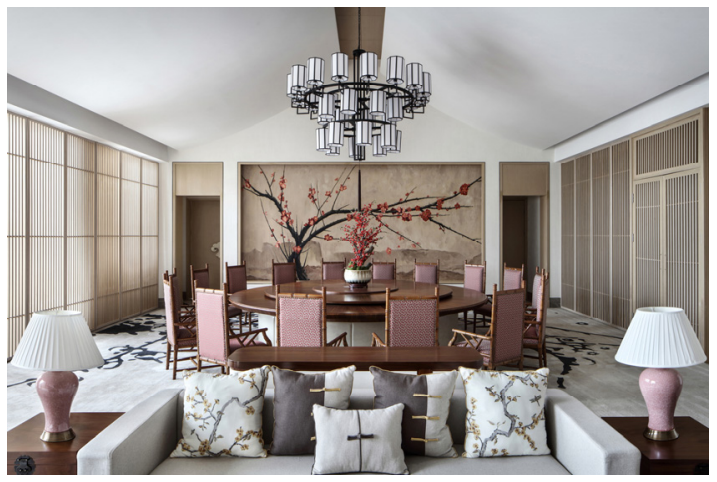

Figure 13. Real view of private room 1.

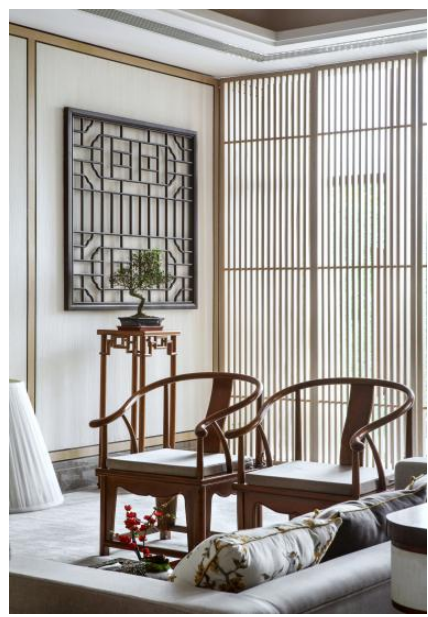

Figure 14. Real view of private room 2.
The design team adopts the original lattice window, plum blossom and other elements, and uses the log tone to create the Zen Oriental concept, "touch the scene to create feelings", and integrate into the quiet realm.

\section{Conclusion}

The only way which must be passed in China's design is orient design, which is based on Oriental culture and philosophy, and integrates with the essence of modern design to make it gain new vitality in the new era. Art categories are integrated with each other, and oriental culture is more extensive and profound. In such a lush and flourishing world, this paper mainly summarizes from three aspects: element minimalism, light and shadow inheritance and meditation practice, and analyzes the practical cases of the team, so as to provide help for other designers. It is hoped that designers can establish cultural confidence based on their understanding of Oriental culture and philosophy, and naturally integrate Oriental Spirit into future design.

\section{References}

1. Zhou, W. Z., (2016) On Oriental design, Famous cities in China 4:4-10.

2. Zhou, W. Z., (2017) Further discussion on Oriental design-The concept, content and research significance of Oriental design, Famous cities in China, 9:4-10.

3. Zhou, W. Z., (2018) Chinese design, more Oriental and more world, People's daily (Overseas Edition), 8:4-7.

4. Li, N., (2012) On the integration of Oriental Culture in modern interior design[J].Modern decoration (Theory), 1:25.

5. Zhou, W. Z., (2019) Third discussion on Oriental design-Analysis of some relations in the construction of Oriental design, Famous cities in China, 1:9-16.

6. Wu, B., (2019) Ben Wu: The inheritance of oriental aesthetics in spatial design, Design, 32(14):88-91.

7. Liu, Y. M., (2021) Discussion on Oriental design aesthetics in the context of localization, Popular literature and art, 5:62-63. 